\title{
Chlorhexidine-Coated CVC-Related Anaphylaxis and Simultaneous Cardiogenic Shock: A Case Report
}

\author{
Hao Tan ${ }^{1}$, Tzu-Ying Li ${ }^{1}$, Kuang-I Cheng ${ }^{1,2}$ and Hung-Te Hsu ${ }^{1,2}$ \\ ${ }^{1}$ Department of Anesthesia, Kaohsiung Medical University Chung-Ho Memorial Hospital, Kaohsiung, Taiwan \\ ${ }^{2}$ Faculty of Anesthesiology, School of Medicine, College of Medicine, Kaohsiung Medical University, Kaohsiung, Taiwan
}

Submission: February 27, 2020; Published: April 04, 2020

*Corresponding author: Hung-Te Hsu, Department of Anesthesiology, Kaohsiung Medical University Hospital, Kaohsiung Medical University, Kaohsiung Taiwan

\section{Abstract}

We present a case who suffered from intraoperative anaphylaxis and simultaneous cardiogenic shock. Through the use of Trans esophageal echocardiography and extracorporeal membrane oxygenation, the patient was successfully resuscitated and discharged without significant sequelae. We hope that the treatment of such patient can be improved with our experience of managing this case.

Keywords: Chlorhexidine; Anaphylaxis; Cardiogenic shock; Resuscitation

Abbreviations: CVC: Central Venous Catheter; BP: Blood Pressure; SPO2: Peripheral Oxygen Saturation; EtCO2: End-Tidal Carbon Dioxide; IV: Intravenous Bolus; CPCR: Cardio-Pulmonary-Cerebral Resuscitation; V-A ECMO: Veno-Arterial Extracorporeal Membrane Oxygenation; ICU: Intensive Care Unit; EKG: Electrocardiogram; VF: Ventricular Fibrillation; DC Defibrillation: Direct-Current Defibrillation

\section{Introduction}

Chlorhexidine is a cationic surfactant synthetic biguanide with broad-spectrum antibacterial and less-pronounced antifungal activity [1,2]. It disrupts microbial cell membranes and coagulates cytoplasmic proteins. Chlorhexidine has a residual activity of several hours, is a nonirritant, nontoxic compound, and works in organic debris. Because of its anti-septic properties, chlorhexidine use is becoming increasingly common in perioperative settings, and with widespread use, increasing numbers of cases of allergy have been reported in the literature [2], including cases of anaphylaxis to chlorhexidine-impregnated devices such as central venous catheters (CVCs). In the past, many similar cases have been reported, and some have even led to life-threatening anaphylaxis. In addition to anaphylaxis, there are other causes of perioperative shock, including sudden reductions in total blood volume through acute blood losses, as in severe hemorrhage; sudden reductions in cardiac output, as in myocardial infarction (heart attack); and widespread dilation of the blood vessels, as in some forms of infection or allergic agent [3]. Herein, we present a patient that sustained anaphylaxis due to the insertion of chlorhexidine-impregnated CVC combined with cardiogenic shock simultaneously. We anticipate that the management experience of this case will help in the treatment of such patients, and also emphasize the importance of cardiac ultrasound in first aid.

\section{Case Report}

A 57-year-old male patient was admitted for colostomy closure. Tracing back his medical history, he had type 2 diabetes mellitus, hypertension and was a hepatitis B carrier. No allergy history was noted. He had received radical proctectomy for rectosigmoid cancer 9 months previously and there was no adverse event during the surgery. After admission, physical examination revealed bilateral clear breathing sound, lab data findings were unremarkable, while pre-operative chest X-ray showed no active cardiopulmonary lesion, but atherosclerotic change of aorta. The pre-operative electrocardiogram (EKG) showed normal sinus rhythm. After recognition of the stability of his vital signs, the patient received Fentanyl $80 \mathrm{mcg}$, Lidocaine $80 \mathrm{mg}$, Propofol $120 \mathrm{mg}$, and Cisatracurium 12mg intravenously for the induction of general anesthesia. Intubation was done smoothly and bilateral breathing sound was clear and symmetrical. Anesthesia was maintained by Sevoflurane. After skin preparation, an 
antimicrobial central venous catheter (CVC) was inserted into his right internal jugular vein. However, tachycardia (heart rate: $110 \mathrm{bpm})$ and desaturation $\left(\mathrm{SpO}_{2}: 89 \%\right)$ were noted 5 minutes later after the insertion. We rechecked the peripheral oxygen saturation in different finger by pulse oximeter but desaturation was still noted. Furthermore, dropping of blood pressure $(90 / 30 \mathrm{mmHg})$ was noted at the same time. Elevated airway pressure was also noted. There was no skin rash or focal edema noted. No antibiotics or other medication was administered before this episode. Ephedrine $12 \mathrm{mg}$ and hydrocortisone $100 \mathrm{mg}$ were injected intravenously, and the central venous catheter was removed. Unfortunately, cardiopulmonary resuscitation was started 5 minutes later due to unobtainable blood pressure and saturation.

The data of arterial gas analysis revealed acidosis $(\mathrm{pH}$ : 7.099) and hypercapnia $\left(\mathrm{PaCO}_{2}: 60 \mathrm{mmHg}\right)$. Hyperkalemia $\left(\mathrm{K}^{+}\right.$: $5.6 \mathrm{mmol} / \mathrm{L}$ ) was also noted, and regular insulin (RI) $8 \mathrm{U}$ along with $50 \%$ dextrose $20 \mathrm{ml}$ was given intravenously. Chest X-ray revealed a wedge-shaped opacity at right middle lobe. Transesophageal echocardiogram (TEE) showed poor cardiac wall motion and flat left ventricle. For aggressive resuscitation and using vasopressor, a non-coated central venous catheter was inserted into the right femoral vein. The cardiac surgeon was consulted for extracorporeal membrane oxygenation (ECMO) installation. After veno-arterial (V-A) ECMO was set, the data of the following arterial gas analysis were improved. The total dosage of epinephrine during cardiopulmonary resuscitation was
$28 \mathrm{mg}$. The operation was cancelled and the patient was sent to the intense care unit (ICU) for further care. The overall resuscitation is stated as Figure 1. After the patient was transferred to the ICU, one episode of ventricular fibrillation (VF) was noted and defibrillation was performed. The EKG reverted to sinus rhythm with V2-3 ST depression. The data of cardiac enzymes were elevated (CPK: 4378, CK-MB: 471.2, Troponin-I: > 80.0). To rule out the possibility of anaphylactic shock, tryptase was checked at the same day. Also, the cardiologist was consulted for further evaluation and management. Coronary angiography was arranged, which revealed right coronary artery (RCA) 100\% stenosis since orifice. Due to unstable hemodynamic status during the procedure, intra-aortic balloon pump (IABP) was used for improving the coronary perfusion. Serial cardiac enzyme data in a week showed downward trend returning to normal range, as shown in Figure 2. Vital signs stabilized and both ECMO and IABP were removed at post-operation day 6 and 7 respectively. Dopamine was used for intermittent hypotension. The patient regained consciousness two days after ECMO removal (post-operation day 8) and his condition improved gradually. Endotracheal tube was removed after smooth respiratory pattern was restored and there was no shortage of breath noted. Immunoglobulin E (IgE) drug hypersensitivity exam showed allergy to the antibiotic Cefaclor, peanuts, soybeans dust mites and animal skin. Tryptase level checked on operation day was elevated $(260 \mu \mathrm{g} / \mathrm{L})$. After a complete course of antibiotics treatment, the patient was discharged and outpatient department follow-up was arranged. The progress of clinical conditions is summarized as Figure 3.

\begin{tabular}{|lll|}
\hline Time & Event & Treatment \\
12:40 & & Induction \\
12:50 & & Antimicrobial CVC insertion \\
13:10 & Unstable vital sign noted: & Recheck monitor $=>\mathrm{BP}: 90 / 30 \mathrm{mmHg}$, \\
& - SpO $_{2}: 89 \%$, HR: $110 \mathrm{bpm}$ & $\mathrm{SpO}_{2}: 70 \%$ \\
& - Elevated airway pressure & Removal of CVC \\
& - EtCO $2: 21 \mathrm{mmHg}$ & Ephedrine $12 \mathrm{mg} \mathrm{IV}$ \\
& & Hydrocortisone $100 \mathrm{mg}$ IV \\
13:15 & Vital signs unobtainable & CPCR started \\
13:25 & Gas analysis: $\mathrm{K}^{+}: 5.6$ & RI $8 \mathrm{U}+50 \%$ dextrose $20 \mathrm{ml}$ \\
13:30 & & Right femoral vein CVC insertion \\
14:00 & & Intraoperative chest X-ray \\
14:25 & & V-A ECMO set \\
& & CPCR end \\
15:30 & & Patient sent to ICU \\
15:38 & EKG: VF noted at ICU & DC defibrillation $200 \mathrm{~J}=>$ sinus rhythm \\
\hline
\end{tabular}

CVC: Central Venous Catheter; BP: Blood Pressure; SPO2: Peripheral Oxygen Saturation; EtCO2: End-Tidal Carbon Dioxide; IV: Intravenous Bolus; CPCR: Cardio-Pulmonary-Cerebral Resuscitation; V-A ECMO: Veno-Arterial Extracorporeal Membrane Oxygenation; ICU: Intensive Care Unit; EKG: Electrocardiogram; VF: Ventricular Fibrillation; DC Defibrillation: Direct-Current Defibrillation Figure 1: Time chart of the operation day. 
Journal of Anesthesia \& Intensive Care Medicine

\begin{tabular}{|l|l|l|l|}
\hline Date and time & CPK (IU/L) & CK-MB $(\mathrm{ng} / \mathrm{ml})$ & Troponin-I $(\mathrm{ng} / \mathrm{ml})$ \\
\hline OP day, 14:28 & 65 & 3.1 & 0.087 \\
\hline Post-OP day 1, 10:21 & 4378 & 471.2 & $>80$ \\
\hline Post-OP day 1, 17:02 & 3759 & 330.9 & $>80$ \\
\hline Post-OP day 1, 20:48 & 2912 & 258.8 & $>80$ \\
\hline Post-OP day 2, 06:08 & 1515 & 90.9 & $>80$ \\
\hline Post-OP day 2, 12:27 & 987 & 51.0 & $>80$ \\
\hline Post-OP day 2, 21:42 & 603 & 24.5 & $>80$ \\
\hline Post-OP day 3, 04:11 & 474 & 13.7 & 80.511 \\
\hline Post-OP day 6, 04:11 & 111 & 1.3 & 16.367 \\
\hline Post-OP day 7, 04:20 & 157 & 1.2 & 8.406 \\
\hline Post-OP day 14, 10:22 & 30 & 3.7 & 0.056 \\
\hline
\end{tabular}

CPK: Creatine Phosphokinase; CK-MB: Creatine Kinase Myocardial Band; OP Day: The Operation Day; Post-OP day: The Day after the Operation

Figure 2: Serial cardiac enzyme data.

\begin{tabular}{|c|c|c|c|}
\hline Date & Day & Event & Treatment \\
\hline $9 / 20$ & OP day & & Consult cardiologist for $\mathrm{r} / \mathrm{o}$ AMI \\
\hline $9 / 21$ & Post-OP day 1 & $\begin{array}{l}\text { Coronary angiography } \\
\text { for } \mathrm{r} / \mathrm{o} \mathrm{AMI}\end{array}$ & $\begin{array}{l}\text { IABP due to unstable blood } \\
\text { pressure }\end{array}$ \\
\hline $9 / 26$ & Post-OP day 6 & & Remove ECMO \\
\hline $9 / 27$ & Post-OP day 7 & & Remove IABP \\
\hline $10 / 01$ & Post-OP day 11 & GCS: E3VeM4 & \\
\hline $10 / 04$ & Post-OP day 14 & Consciousness clear & Extubation \\
\hline $10 / 27$ & Post-OP day 37 & & Discharged and OPD follow-up \\
\hline
\end{tabular}

OP day: The Operation day; Post-OP day: The day after the operation; AMI: Acute Myocardial Infarction; IABP: Intra-Aortic Balloon Pump; ECMO: Extracorporeal Membrane Oxygenation; GCS: Glasgow Coma Scale; OPD: Out-Patient Department

Figure 3: Time chart of clinical presentation.

\section{Discussion}

Herein, we presented a case with unstable hemodynamic status after insertion of an antibiotic-coated CVC on the right side of the neck. Elevated airway resistance indicating the possibility of bronchospasm [4-10] as well as the following lab exam showing elevated Tryptase level [10-13] all contributed to the impression of anaphylaxis. However, according to the medical record, the same medications were used for anesthesia induction and maintenance when the patient underwent surgery of radical proctectomy. No antibiotics or other medications were given to the patient before this episode. On the other hand, there were no notable EKG ischemia changes such as T wave inversion, ST depression or ST elevation before the resuscitation. Pre-operative EKG showed normal sinus rhythm, but there was one episode of ventricular fibrillation after the patient was sent to the ICU. In addition, during the resuscitation, the images of TEE showed poor heart wall motion and flattened left ventricle; furthermore, coronary angiography showed RCA $100 \%$ occlusion. The patient denied any cardiac disease history during the anesthesia consultation. However, he had a history of hypertension, and chest X-ray showed atherosclerosis of the aorta, which might have increased the risk for cardiovascular events including thromboembolism. The initial presentation of unstable vital signs was tachycardia with mildly dropped blood pressure, along with desaturation and elevated airway pressure. All of the above symptoms and signs may be seen in a patient with anaphylaxis [4-10]. Though there was no skin rash noted throughout the resuscitation, it is possible that cutaneous vasodilation was absent at the early stage of anaphylaxis. To maintain hemodynamic stability, the sympathetic nervous system may be activated, which induces peripheral vasoconstriction [5,14]. The same medications (Fentanyl, Lidocaine, Propofol, Cisatracurium and Sevoflurane) were used for anesthesia induction and maintenance during the last surgery the 
patient had received; additionally, no other medication was given to the patient before hemodynamic collapse, so it is likely that the inserted antimicrobial CVC triggered the anaphylaxis reaction and induced hemodynamic instability, if the unstable vital signs are attributed to allergic reaction. Although anaphylactic shock can trigger unstable vital signs, so can cardiogenic shock [3]. During resuscitation, the TEE exam revealed poor cardiac wall motion. In addition, the coronary angiography showed RCA total occlusion, which might have been the cause of the myocardial ischemia. However, it is also possible that the poor cardiac contractility was caused by worsened myocardial perfusion due to unstable blood pressure $[5,6,10,11]$. Therefore, if a patient were to develop anaphylactic shock, early TEE examination would be very helpful in detecting whether there is simultaneous myocardial ischemia. The additional cardiac support might have helped in maintaining hemodynamic instability during resuscitation, and the use of ECMO may have assisted the maintenance of cardiac output and thus systemic perfusion, which improved the quality of resuscitation [15]. There are two types of ECMO: venovenous $(\mathrm{V}-\mathrm{V})$ and venoarterial (V-A). V-V configuration is mainly used in patients with respiratory failure and normal heart function, whereas V-A configuration is used in patients who need cardiac support [16]. In our case, V-A ECMO was the choice due to TEE revealing poor cardiac function. Fortunately, the patient's condition improved, and the ECMO was removed at postoperative day 6.

\section{Conclusion}

We present a case suffering from Chlorhexidine-coated CVCrelated anaphylaxis and simultaneous cardiogenic shock. Though it might be difficult in diagnosing the predominating cause, TEE exam may be helpful in early cardiac evaluation. In addition to standard resuscitation, the use of ECMO might help maintain adequate cardiac output and systemic perfusion, thus increasing the quality of resuscitation and improving patient outcome.

References

1. Opstrup MS, Jemec G, Garvey, LH (2019) Chlorhexidine Allergy: On the Rise and Often Overlooked. Curr Allergy Asthma Rep 19(5): 23.

2. Rose MA, Garcez T, Savic S, Garvey LH (2019) Chlorhexidine allergy in the perioperative setting: a narrative review. Br J Anaesth 123(1): 95-103.
3. Morozowich ST, Ramakrishna H (2015) Pharmacologic agents for acute hemodynamic instability: recent advances in the management of perioperative shock- a systematic review. Ann Card Anaesth 18(4): 543-554.

4. Berrío Valencia MI (2015) Perioperative anaphylaxis. Brazilian journal of anesthesiology 65(4): 292-297.

5. Dewachter P, Mouton-Faivre C, Hepner DL (2015) Perioperative anaphylaxis: what should be known? Curr Allergy Asthma Rep 15(5): 21.

6. Ebo DG, Clarke R C, Mertes PM, Platt PR, Sabato V, etal. (2019) Molecular mechanisms and pathophysiology of perioperative hypersensitivity and anaphylaxis: a narrative review. Br J Anaesth 123(1): 38-49.

7. Justiz Vaillant AA, Zito PM (2020) Immediate Hypersensitivity Reactions. In StatPearls.

8. Kannan JA, Bernstein JA (2015) Perioperative anaphylaxis: diagnosis, evaluation, and management. Immunol Allergy Clin North Am 35(2): 321-334.

9. Laguna JJ, Archilla J, Doña I, Corominas M, Gastaminza G, et al. (2018) Practical Guidelines for Perioperative Hypersensitivity Reactions. J Investig Allergol Clin Immunol 28(4): 216-232.

10. LoVerde D, Iweala OI, Eginli A, Krishnaswamy G (2018) Anaphylaxis. Chest 153(2): 528-543.

11. Jimenez-Rodriguez TW, Garcia-Neuer M, Alenazy LA, Castells M (2018) Anaphylaxis in the 21st century: phenotypes, endotypes, and biomarkers. J Asthma Allergy 11: 121-142.

12. Montañez MI, Mayorga C, Bogas G, Barrionuevo E, FernandezSantamaria R, et al. (2017) Epidemiology, Mechanisms, and Diagnosis of Drug-Induced Anaphylaxis. Front Immunol 8: 614.

13. Sala-Cunill A, Guilarte M, Cardona V (2018) Phenotypes, endotypes and biomarkers in anaphylaxis: current insights. Curr Opin Allergy Clin Immunol 18(5): 370-376.

14. Garvey LH, Dewachter P, Hepner, DL, Mertes PM, Voltolini S, et al. (2019) Management of suspected immediate perioperative allergic reactions: an international overview and consensus recommendations. Br J Anaesth 123(1): 50-64.

15. Kim SJ, Kim HJ, Lee HY, Ahn HS, Lee SW (2016) Comparing extracorporeal cardiopulmonary resuscitation with conventional cardiopulmonary resuscitation: A meta-analysis. Resuscitation 103: 106-116.

16. Tan BK (2017) Extracorporeal membrane oxygenation in cardiac arrest. Singapore medical journal 58(7): 446-448. 
This work is licensed under Creative Commons Attribution 4.0 License DOI: $10.19080 / J A I C M .2020 .10 .555795$
Your next submission with Juniper Publishers will reach you the below assets

- Quality Editorial service

- Swift Peer Review

- Reprints availability

- E-prints Service

- Manuscript Podcast for convenient understanding

- Global attainment for your research

- Manuscript accessibility in different formats ( Pdf, E-pub, Full Text, Audio)

- Unceasing customer service

Track the below URL for one-step submission https://juniperpublishers.com/online-submission.php 